\section{BMJ Open Respiratory Research}

\title{
Initiation, scale-up and outcomes of the Cambodian National MDR-TB programme 2006-2016: hospital and community-based treatment through an NGO-NTP partnership
}

Sophan Sam, ${ }^{1}$ Adrienne E Shapiro, ${ }^{1,2}$ Thim Sok, ${ }^{1}$ Sokhan Khann, ${ }^{1,3}$ Rassi So, ${ }^{1}$ Sopheap Khem, ${ }^{1}$ Sokhem Chhun, ${ }^{1}$ Sarith Noun, ${ }^{1}$ Bonamy Koy, ${ }^{4}$ Prum Chhom Sayouen, ${ }^{4}$ Chun Im Sin, ${ }^{5}$ Heng Bunsieth, ${ }^{1}$ Tan Eang Mao, ${ }^{4}$ Anne E Goldfeld ${ }^{1,6}$

To cite: Sam S, Shapiro AE, Sok T, et al. Initiation, scaleup and outcomes of the Cambodian National MDR-TB programme 2006-2016: hospital and communitybased treatment through an NGO-NTP partnership. BMJ Open Resp Res 2018;5:e000256. doi:10.1136/ bmjresp-2017-000256

SS, AES and TS contributed equally.

Received 25 October 2017 Revised 21 February 2018

\section{Check for updates}

${ }^{1}$ Cambodian Health Committee, Phnom Penh, Cambodia

2Department of Medicine, University of Washington, Seattle, Washington, USA ${ }^{3}$ WHO-Cambodia, Phnom Penh, Cambodia

${ }^{4}$ National Center for Tuberculosis and Leprosy Control, Phnom Penh, Cambodia

${ }^{5}$ Khmer Soviet Friendship Hospital, Phnom Penh,

Cambodia

${ }^{6}$ Program in Cellular and Molecular Medicine, Children's Hospital Boston and Harvard Medical School, Boston, Massachusetts, USA

Correspondence to Dr Anne E Goldfeld; anne.goldfeld@childrens. harvard.edu

\section{ABSTRACT}

Introduction Prolonged inpatient multidrug-resistant tuberculosis (MDR-TB) treatment for all patients is not sustainable for high-burden settings, but there is limited information on community-based treatment programme outcomes for MDR-TB.

Methods The Cambodian Health Committee, a nongovernmental organisation (NGO), launched the Cambodian MDR-TB programme in 2006 in cooperation with the National Tuberculosis Program (NTP) including a community-based treatment option as a key programme component. The programme was transferred to NTP oversight in 2011 with NGO clinical management continuing. Patients electing to receive home-based treatment were followed by a dedicated adherence supporter and a multidisciplinary outpatient team of nurses, physicians and community health workers. Patients hospitalised for $>1$ month of treatment (hospital based) received similar management after discharge. All patients received a standardised second-line MDR-TB regimen and were provided nutritional and adherence support. Outcomes were reviewed for patients completing 24 months of treatment and predictors of treatment success were evaluated using logistic regression.

Results 0 f 582 patients with MDR-TB who initiated treatment between September 2006 and June 2016, 20\% were HIV coinfected, 288 (49\%) initiated community-based treatment and $294(51 \%)$ received hospital-based treatment. Of 486 patients with outcomes available, $364(75 \%)$ were cured, $10(2 \%)$ completed, 28 (6\%) were lost to follow-up, $3(0.6 \%)$ failed and $77(16 \%)$ died. There was no difference between treatment success in community versus hospitalbased groups (adjusted OR (aOR) 1.0, p=0.99). HIV infection, older age and body mass index $<16$ were strongly associated with decreased treatment success (aOR 0.33, $p<0.001$; aOR 0.40, $p<0.001$; a0R 0.40; $p<0.001$ ).

Conclusions Cambodia's NGO-NTP partnership successfully developed and scaled up a model MDR-TB treatment programme. The first large-scale MDR-TB programme in Asia with a significant community-based component, the programme achieved equally high treatment success in patients with community-based compared with hospitalbased initiation of MDR treatment.

\section{INTRODUCTION}

Cambodia has a tuberculosis (TB) incidence of 345/100 000 annually, the 15th highest incidence among the WHO-designated 30 highest burden countries globally. It is estimated that $1.8 \%$ of new $\mathrm{TB}$ cases in Cambodia and $11 \%$ of retreatment TB cases are multidrug resistant (MDR)/rifampicin resistant, ${ }^{2}$ indicating ongoing need to improve the diagnosis and treatment of both drug-susceptible and drug-resistant (DR) TB to prevent a further increase in the burden of multidrug-resistant tuberculosis (MDR-TB). Regional neighbours Myanmar and Vietnam are designated as high MDR burden countries, ${ }^{3}$ underscoring the need for innovative regional approaches to address the epidemic. Furthermore, over the last decade, Cambodia has been a high-HIV/TB burden country, with over $10 \%$ of patients with $\mathrm{TB}$ coinfected with HIV, and laboratory surveillance identifying MDR-TB in $5 \%$ of all HIV-associated TB. ${ }^{45}$

MDR-TB is typically treated in an inpatient setting for the first 6-8 months of treatment. The requirement for long-term hospitalisation in an airborne isolation setting places a tremendous burden on both the patient and the health system, ${ }^{67}$ particularly in resource-limited settings where bed availability limits access to treatment. In Cambodia, there are currently 65 isolation beds, but in 2015 alone there were an estimated 570 MDR cases requiring treatment. ${ }^{2}$ As detection of MDR-TB continues to improve, the capacity to meet the treatment needs requires alternate approaches. We note that there is limited information on 
the outcomes of community-based MDR-TB treatment compared with hospital-based MDR-TB treatment.

The Cambodian Health Committee (CHC) is a non-governmental organisation (NGO) providing comprehensive TB and HIV treatment and poverty reduction interventions in Cambodia, ${ }^{8}$ including community-based directly observed therapy (DOTS) for drug-susceptible TB in Cambodia beginning in $1994,{ }^{9}$ which was adopted as the national protocol in $2004 .{ }^{10}$ In 2006, CHC adapted this community-based treatment model to provide care and support for persons with MDR-TB, the first MDR-TB treatment programme in Cambodia. CHC offered eligible patients the option to initiate or continue treatment as outpatients early in the course of treatment, and to receive home-based care with the support of community-based health staff. We report here the overall treatment outcomes of the Cambodian MDR-TB programme for patients initiated on treatment from 2006 to June 2015. We also compare the treatment success between patients treated with an extended inpatient initiation phase versus community-based treatment beginning within the first month of treatment initiation.

\section{STUDY POPULATION AND METHODS \\ Setting \\ Programme origins}

Prior to 2006, there was no mechanism for public sector MDR-TB testing or treatment in Cambodia (figure 1). The CHC applied to the WHO Green Light Committee (GLC) in 2006 on behalf of patients enrolled in the CAMbodian Early vs. Late Initiation of Antiretrovirals (CAMELIA) trial ${ }^{11}$ testing optimal timing of HIV treatment in immunosuppressed patients with $\mathrm{TB}$, including 13 found to have MDR-TB through TB culture and drug resistance testing performed per trial protocol. CHC received GLC approval in 2007 for a second application with the National Tuberculosis Program (NTP) to extend MDR treatment to new patients outside the CAMELIA study. ${ }^{12}$ CHC then established MDR treatment sites throughout the country in partnership with the NTP,

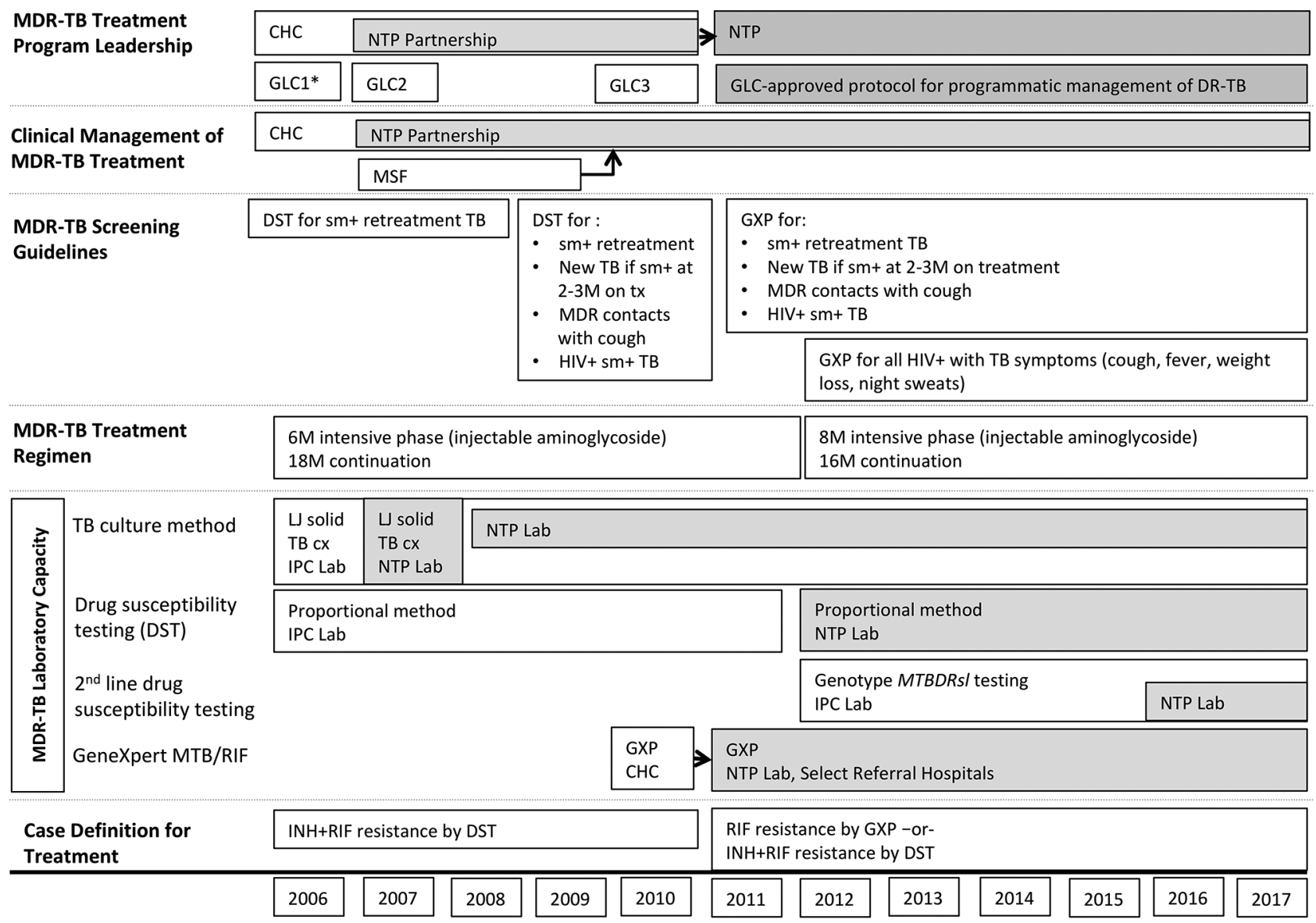

Figure 1 Timeline of development of key elements of the Cambodian MDR-TB treatment programme. *GLC1 application for patients enrolled in the CAMbodian Early vs. Late Introduction of Antiretrovirals (CAMELIA) clinical trial only. CHC, Cambodian Health Committee; cx, culture; GLC, Green Light Committee of the WHO for MDR-TB Treatment; GXP, GeneXpert MTB/RIF; INH, isoniazid; IPC, Institut Pasteur du Cambodge; LJ, Lowenstein-Jensen solid culture method; MDR-TB, multidrug-resistant tuberculosis; MSF, Medecins Sans Frontieres Belgium and France programmes; NTP, National Tuberculosis Program; RIF, rifampicin; sm+, sputum smear positive. 
initially establishing three hospitals as treatment centres and constructing and renovating 29 isolation rooms at these sites. As of 2017 there were 65 isolation rooms in 11 referral hospitals throughout Cambodia. A third successful CHC application to the GLC in 2010 further expanded and began national scale-up of DR-TB treatment. In 2011, the NTP assumed operational leadership of the MDR-TB programme to create the national MDR-TB treatment programme in partnership with CHC, with the support of the WHO. The NTP is now responsible for overall programmatic management, including screening suspected MDR cases for drug resistance and baseline contact screening. CHC continues to provide essential programme services including patient follow-up, treatment support and education, clinical follow-up of patients, side effect management, home infection control and reporting, as well as technical assistance to the NTP.

\section{Patient screening and identification}

Prior to 2011, MDR-TB cases were detected through drug-susceptibility testing (DST) of smear-positive retreatment TB cases. Patients were notified to the CHC through a combination of CHC staff examining provincial clinic TB registers as well as NTP staff contacting CHC when such patients were identified. Screening criteria were expanded to include: (1) all pulmonary retreatment cases; (2) patients with TB who are persistently smear positive after months 2-3 of TB treatment; (3) close contacts of MDR-TB cases with cough $>2$ weeks; and (4) HIV-infected smear-positive patients with TB. ${ }^{13}$ HIV counselling and testing was performed at the time of TB diagnosis. In 2012, the screening algorithm was revised to recommend screening any HIV-infected patient with signs/symptoms of TB (cough, weight loss, fever, night sweats) using GeneXpert MTB/RIF (GXP).

Medecins Sans Frontieres (MSF) Belgium and MSF France programmes provided MDR-TB treatment in two provinces between 2007 and 2009. Patients who were still on treatment $(n=28)$ when MSF withdrew from this sector were transferred to the $\mathrm{CHC}$ programme and are included in this analysis.

\section{Laboratory diagnosis and follow-up}

Initially, in 2006, Lowenstein-Jensen culture and DST by the proportional method were performed at the Institut Pasteur du Cambodge (IPC), in a laboratory externally validated by the College of American Pathologists and the Japanese Supranational Reference Laboratory. In 2007, solid TB culture became available at the NTP laboratory, and in 2008 liquid culture was introduced. DST was thereafter mainly conducted in the national laboratory beginning in 2011. Second-line drug susceptibility testing has been performed at IPC using GenoType MTBDRsl since 2012. The CHC obtained the first GXP platform in Asia in 2010 for detection of Mycobacterium tuberculosis and rifampin (RIF) resistance through a research protocol. ${ }^{14}$
Box 1 Standardised multidrug-resistant tuberculosis (MDRTB) treatment regimen

Intensive phase: 6-8 months
Injectable aminoglycoside (kanamycin, amikacin or capreomycin).
Fluoroquinolone (ofloxacin, levofloxacin or moxifloxacin).
Ethionamide.
Pyrazinamide.
Cycloserine or para-aminosalicylic acid (PAS).
If sensitive, ethambutol was also added.
Continuation phase: $\mathbf{1 8 \text { months }}$
Levofloxacin/moxifloxacin.
- Ethionamide.
Cycloserine/PAS.
Pyrazinamide.
Ethambutol, if sensitive.

This GXP platform was then transferred to the NTP for use for public sector MDR testing. The NTP subsequently acquired additional GXP platforms, and in 2011, an MDR screening algorithm was adopted to use GXP as the initial screening test for suspected MDR. ${ }^{15}$

\section{Diagnostic criteria}

MDR was defined as infection with $M$. tuberculosis resistant to isoniazid (INH) and RIF as detected by DST. Patients were empirically started on MDR treatment on the basis of strong clinical suspicion or, starting in 2011, GXP evidence of RIF resistance. If DST detected first-line drug resistance not meeting criteria for MDR, the treatment regimen and duration was modified accordingly. TB with GXP-detected RIF resistance for which DST or genetic resistance testing was not available was treated presumptively as MDR.

\section{Treatment protocol and programme components}

The MDR-TB treatment regimen in Cambodia consisted of a standardised regimen (box 1). If a patient had culture-positive MDR-TB or no clinical improvement at 4 months, second-line DST was performed and the regimen was modified to include only susceptible drugs. In 2012, the injection phase of treatment was extended to 8 months for all patients based on WHO recommendations. ${ }^{16}$

As part of the initiation of the countrywide MDR programme, MDR Management Committees (MMC) were established at each provincial hospital designated as an MDR referral centre in order to safely expand treatment initiation capacity. MMCs included a government physician, a CHC physician, a nurse with expertise in MDR and/or a radiologist at the site. The MMC reviewed laboratory, clinical and imaging data for each patient prior to initiating treatment and agreed on the appropriate treatment profile and dosing for the patient. 


\section{Community versus hospital-based care}

Patients without hospital-level clinical requirements for managing comorbidities, available home-based support and preference for outpatient care could elect to start treatment as outpatients without hospital admission. The community-based treatment group was defined as patients who initiated treatment in the community or who were discharged from the hospital less than 30 days after treatment initiation (community arm). Patients who were hospitalised for 30 days or more, or who died before discharge were considered to be in the hospital-based treatment group (hospitalised arm). The decision to discharge a patient home was made in consultation with the patient, the inpatient clinicians and the community care team.

At the time of initiation of outpatient treatment, a treatment contract was signed by the patient, a treatment supporter (usually a nurse) from the health centre closest to the patient, a second treatment supporter designated by the patient and CHC staff. The treatment supporters supervised daily medication administration, provided motivation, maintained awareness of side effects and contacted the health centre or CHC staff in the event of clinical deterioration. Local health centre staff administered daily injectable medications in the intensive phase of MDR-TB therapy, monitored adherence during this phase and reported any clinical problems to the CHC outpatient monitoring team by phone.

For patients returning home prior to sputum culture conversion, education and supplies were provided for home-based infection control, including masks for patients and family members, recommendations for maintaining adequate ventilation and isolating small children from the patient. Assistance with creating an adjoining shelter for the patient was also provided if there were inadequate existing ventilated space for the patient. Symptom screening of household contacts was conducted at monthly visits. Contacts suspected to have TB had sputum collected by the visiting staff on the spot or were directed to the local health centre for screening. Direct and financial support was provided for nutrition (including baby formula for patients who could not breast feed due to treatment toxicity) and transportation for laboratory tests and hospital visits.

Patients with MDR-TB with HIV infection were referred to local centres providing antiretroviral therapy (ART) for clinical follow-up. MDR-TB clinicians worked closely with ART providers to monitor combined treatments, side effects and dose adjustments.

Ongoing education and training in MDR diagnosis, treatment and clinical follow-up including side effect management was provided by CHC to clinicians at the regional treatment sites and to local health staff. CHC designed and implemented nationwide training for the NTP in 2006 on MDR-TB diagnosis and treatment and continues to provide ongoing clinical mentoring and care of patients countrywide.

\section{Patient follow-up and monitoring}

The CHC mobile outpatient monitoring team of doctors and/or nurses and local government health staff made visits to patient homes monthly to resupply medication, screen for side effects, monitor clinical progress and collect sputum for treatment monitoring. Visits were more frequent if clinically indicated for adverse events or worsening of clinical symptoms. A CHC physician made periodic home visits to patients as necessary. CHC field nurses set up community care by linking the patient to the local health centre and village health support group in their village and trained the treatment supporter. Any clinical or adherence issues were reported to the CHC team for problem solving. Patients visited the health centre quarterly for physical examination. Any treatment or adherence issues that could not be readily solved were reported to the CHC monitoring team by local government health centre staff.

Treatment outcomes were defined and reported according to WHO definitions for MDR-TB. ${ }^{17}$ Outcomes for the cohort are reported for patients initiating therapy by June 2015 who therefore had the opportunity to complete 24 months of follow-up by the time of data censoring in June 2017. For analyses of associations with treatment outcome, we defined a combined outcome of 'treatment success', defined as either 'cure' or 'completion'. Outcomes of 'died', 'lost to follow-up' or 'failed' were defined as 'no treatment success'. Patients who transferred out were excluded from this categorisation scheme as their outcomes were unknown.

\section{Statistical analysis}

Demographic, clinical, laboratory and microbiological data were routinely abstracted from patient records into a clinical database maintained by the CHC. An anonymised subset of this database was entered into a Microsoft Excel spreadsheet with range and error-checking formulas. Only the first episode of treatment for MDR-TB was included in this analysis for each person. Statistical analyses were conducted using Stata (College Station, TX). Descriptive frequencies were calculated and compared using $\mathrm{X}^{2}$ tests. Medians of continuous variables were compared using the Mann-Whitney test. Predictors of treatment success were assessed using bivariate and multivariate logistic regression. Factors of a priori interest were included in the multivariable model.

\section{Ethics}

As this study was based on existing programme records collected as part of routine clinical care, and no patient identifying information was collected, ethical clearance was not required according to local regulations.

\section{RESULTS}

Between September 2006 and June 2016, CHC and the NTP screened 9423 patients and persons at risk of MDR 


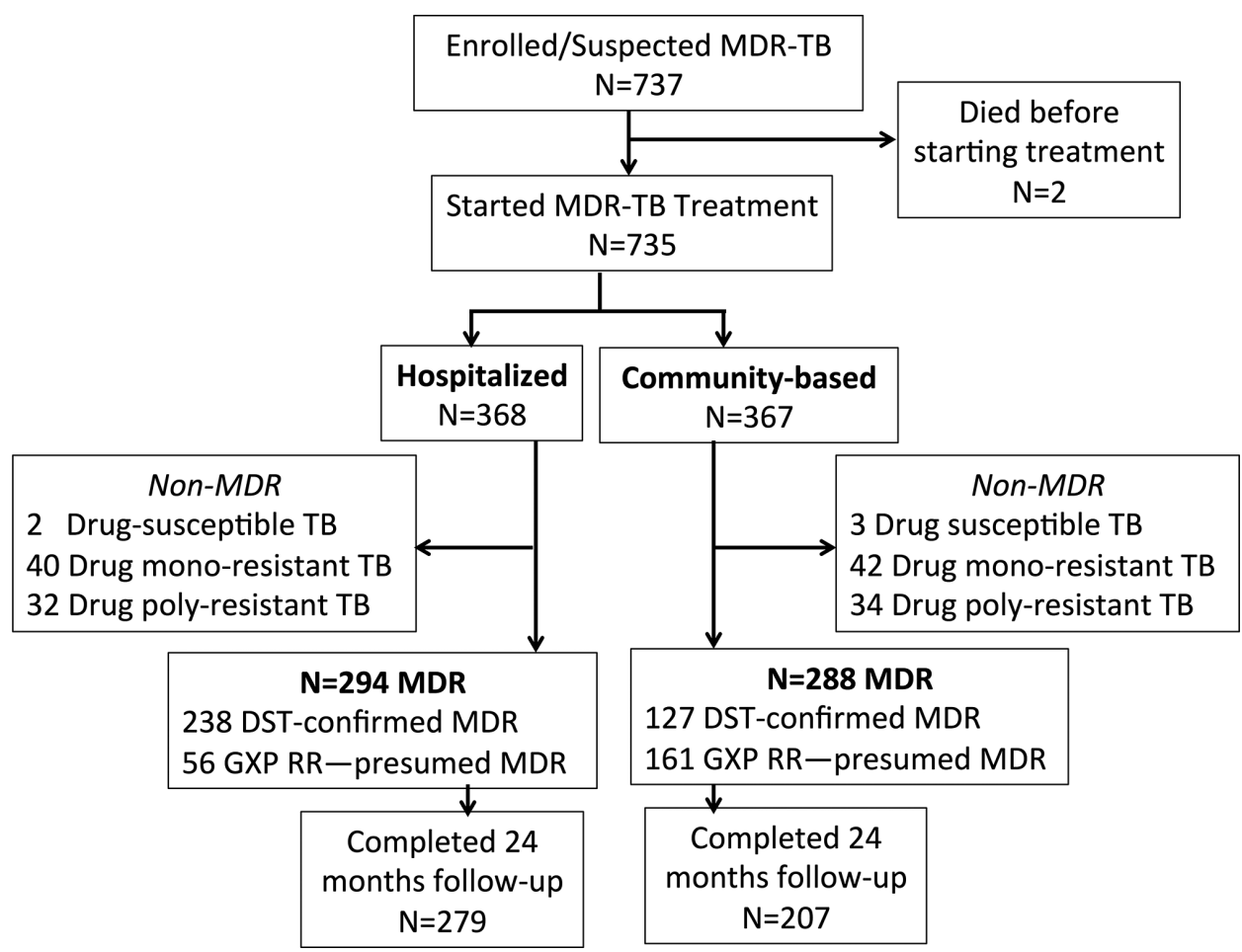

Figure 2 MDR treatment enrolment and diagnoses, 2006-2016. DST, drug-susceptibility testing; GXP, GeneXpert MTB/RIF; MDR-TB, multidrug-resistant tuberculosis; RR, rifampin resistant.

using DST, by GXP and/or DST from December 2011 onwards. A total of 737 patients were enrolled in the CHC MDR treatment programme (figure 2). Two patients died before initiating treatment. A total of 735 initiated MDR treatment. Of these patients, 365 were confirmed by DST to have definitive MDR, and 217 were considered to be MDR based on GXP results without DST confirmation available. DST determined that 198 patients initiated on MDR treatment had DR-TB not meeting criteria for MDR (INH monoresistance, RIF monoresistance or polydrug resistance). Up to 288 (49\%) of MDR patients were treated in the community arm, including two patients who were screened, initiated on treatment and followed while in prison but were not hospitalised at the start of treatment. The hospitalised treatment group consisted of $294(51 \%)$ patients.

\section{Patient demographics}

Baseline characteristics were similar between the community and hospitalised treatment groups (table 1). The median duration of hospitalisation for community patients was 6 days (IQR 0-12 days) and for hospitalised patients was 84 days (IQR 45-180 days). There were more than twice as many men as women $(63 \%$ male vs $37 \%$ female) in the cohort overall, with women making up a greater proportion of the community patients $(41 \%$ vs $33 \%, \mathrm{p}=0.046)$. The age distribution was similar between community and hospitalised groups; the median age at the time of starting MDR treatment was 44 (IQR 33-55), including three children younger than 15 years and 48 adults older than 65 years. HIV infection was similar in the two groups and present in $117(20 \%)$ of patients initiating MDR treatment, with 110 (94\%) taking ART at or within 4 weeks of MDR treatment initiation. Patients were severely underweight with a median body mass index (BMI) of 17.2 (IQR 15.2-19). BMI was lower in the hospitalised group (16.9 vs $17.4, \mathrm{p}=0.04$ ). The hospitalised group had greater overall drug resistance compared with the community group ( $57 \%$ with additional resistance beyond INH and RIF vs $30 \%, \mathrm{p}<0.001)$. There were four cases of extensively drug-resistant (XDR) TB identified and confirmed by second-line drug testing and one case of pre-XDR-TB. A history of prior TB treatment was present in $306(53 \%)$ of patients initiated on MDR-TB treatment; $276(47 \%)$ were being treated for their first episode of TB, and this did not differ between community and hospitalised groups.

\section{Treatment outcomes}

Of 486 patients initiated on MDR-TB treatment, 374 $(77 \%)$ had treatment success, with 28 (6\%) lost to follow-up, $3(0.6 \%)$ failed and $77(16 \%)$ died (table 2$)$. The proportion of each outcome did not differ significantly between patients in the community and hospitalised groups $(\mathrm{p}=0.46)$. One patient was lost to follow-up, was subsequently re-enrolled after 2 years and treatment was ongoing at the time of data censoring. Among the 117 HIV-coinfected patients, $65(65 \%)$ had treatment success, $32(27 \%)$ died, $1(1 \%)$ failed and $8(7 \%)$ lost to follow-up, and $11(9 \%)$ continued on treatment at the time of data censoring. 
Table 1 Demographics and baseline clinical characteristics of patients with MDR-TB, 2006-2016. Percentages may add up to $>100 \%$ due to rounding

\begin{tabular}{|c|c|c|c|c|}
\hline & $\begin{array}{l}\text { Total } \\
\text { n (\%) }\end{array}$ & $\begin{array}{l}\text { Community based } \\
\mathrm{n}(\%)\end{array}$ & $\begin{array}{l}\text { Hospital based } \\
\text { n (\%) }\end{array}$ & $P$ values \\
\hline & 582 & $288(49)$ & $294(51)$ & \\
\hline \multicolumn{5}{|l|}{ Sex } \\
\hline Female & $213(37)$ & $117(41)$ & $96(33)$ & \\
\hline Male & $369(63)$ & $171(59)$ & $198(67)$ & 0.046 \\
\hline \multicolumn{5}{|l|}{ Age } \\
\hline Median (IQR) & $45(33-55)$ & $45(33-56)$ & $45(34-53)$ & 0.52 \\
\hline$<15$ & $3(0.5)$ & $1(0.4)$ & $2(0.7)$ & \\
\hline $15-29$ & $91(16)$ & $44(15)$ & $47(16)$ & \\
\hline $30-49$ & $267(46)$ & $128(44)$ & $139(47)$ & \\
\hline $50+$ & $221(38)$ & $115(40)$ & $106(36)$ & 0.76 \\
\hline \multicolumn{5}{|l|}{ HIV status } \\
\hline Positive & $117(20)$ & $53(18)$ & $64(22)$ & \\
\hline Negative & $465(80)$ & $235(82)$ & $230(78)$ & 0.31 \\
\hline \multicolumn{5}{|l|}{ ART (HIV+ only) } \\
\hline Yes & $110(94)$ & $50(94)$ & $60(94)$ & \\
\hline No & 7 (6) & $3(6)$ & $4(6)$ & 0.89 \\
\hline Median BMI (IQR) & $17.2(15.2-19)$ & $17.4(15.6-19.4)$ & $16.9(15-18.8)$ & 0.04 \\
\hline \multicolumn{5}{|l|}{ Resistance pattern } \\
\hline GXP only (RIF) & $217(37)$ & $161(56)$ & $56(19)$ & \\
\hline $\mathrm{INH}+\mathrm{RIF}$ only & $108(19)$ & $37(13)$ & $71(24)$ & \\
\hline $\mathrm{INH}+\mathrm{RIF}+$ & $253(43)$ & $87(30)$ & $166(57)$ & \\
\hline XDR & $4(0.7)$ & $3(1)$ & $1(0.3)$ & $<0.001$ \\
\hline \multicolumn{5}{|l|}{ TB history } \\
\hline No prior TB treatment & $276(47)$ & $146(51)$ & $130(44)$ & \\
\hline Prior TB treatment & $306(53)$ & $142(49)$ & $164(56)$ & 0.11 \\
\hline \multicolumn{5}{|l|}{ Site of TB } \\
\hline Pulmonary & $562(97)$ & $282(98)$ & 280 (95) & \\
\hline Extrapulmonary & $16(3)$ & 3 (1) & $13(4)$ & \\
\hline Pulmonary and extrapulmonary & $4(0.7)$ & $3(1)$ & $1(0.3)$ & 0.03 \\
\hline
\end{tabular}

ART, antiretroviral therapy; BMI, body mass index; GXP, GeneXpert MTB/RIF; INH, isoniazid; INH+RIF+, additional drug resistance beyond INH and RIF, not XDR; MDR-TB, multidrug-resistant tuberculosis; RIF, rifampicin; XDR, extensively drug-resistant TB.

Table 2 Treatment outcomes of patients with MDR-TB, 2006 to June 2015

\begin{tabular}{|c|c|c|c|c|}
\hline & $\begin{array}{l}\text { Total }(n=486) \\
n(\%)\end{array}$ & $\begin{array}{l}\text { Community based } \\
(n=207) \\
n(\%)\end{array}$ & $\begin{array}{l}\text { Hospital based }(n=279) \\
n(\%)\end{array}$ & $P$ values \\
\hline Treatment success & $374(77)$ & $160(77)$ & $259(77)$ & \\
\hline Cured & $364(75)$ & $155(75)$ & $209(75)$ & \\
\hline Completed & $10(2)$ & $5(2)$ & $5(2)$ & \\
\hline Lost to follow-up & $28(6)$ & $15(7)$ & $13(5)$ & \\
\hline Failed & $3(0.6)$ & $1(0.5)$ & $2(0.7)$ & \\
\hline Died & $77(16)$ & $28(14)$ & $49(18)$ & \\
\hline Transfer out & $4(0.8)$ & $3(1)$ & $1(0.4)$ & 0.46 \\
\hline
\end{tabular}

MDR-TB, multidrug-resistant tuberculosis. 


\begin{tabular}{|c|c|c|c|c|}
\hline & OR $(95 \% \mathrm{Cl})$ & $P$ values & Adjusted OR (95\% Cl) & $P$ values \\
\hline \multicolumn{5}{|l|}{ Treatment group } \\
\hline Hospitalised & 1.0 & & 1.0 & \\
\hline Community & $1.09(0.70$ to 1.68$)$ & 0.71 & $1.0(0.63$ to 1.60$)$ & 0.99 \\
\hline \multicolumn{5}{|l|}{ Sex } \\
\hline Female & 1.0 & & 1.0 & \\
\hline Male & $0.80(0.51$ to 1.27$)$ & 0.35 & 0.67 (0.41 to 1.09$)$ & 0.11 \\
\hline \multicolumn{5}{|l|}{ Age } \\
\hline$<44$ & 1.0 & & 1.0 & \\
\hline$\geq 44$ & $0.53(0.34$ to 0.83$)$ & 0.006 & 0.40 (0.24 to 0.67$)$ & $<0.001$ \\
\hline \multicolumn{5}{|l|}{ Resistance pattern } \\
\hline INH+RIF or GXP/RIF & 1.0 & & 1.0 & \\
\hline $\mathrm{INH}+\mathrm{RIF}+$ & 1.20 (0.78 to 1.85$)$ & 0.41 & 1.15 (0.73 to 1.83$)$ & 0.54 \\
\hline XDR & 0.31 (0.04 to 2.27$)$ & 0.25 & 0.28 (0.04 to 2.23 ) & 0.23 \\
\hline \multicolumn{5}{|l|}{ HIV } \\
\hline Negative & 1.0 & & 1.0 & \\
\hline Positive & $0.47(0.29$ to 0.78$)$ & 0.003 & 0.33 (0.19 to 0.64$)$ & $<0.001$ \\
\hline \multicolumn{5}{|l|}{ Baseline BMI } \\
\hline$\geq 16$ & 1.0 & & 1.0 & \\
\hline$<16$ & 0.41 (0.26 to 0.64$)$ & $<0.001$ & $0.40(0.25$ to 0.64$)$ & $<0.001$ \\
\hline
\end{tabular}

BMI, body mass index; GXP, GeneXpert MTB/RIF; INH, isoniazid; INH+RIF+, additional drug resistance beyond INH and RIF, not XDR; MDR, multidrug resistant; RIF, rifampicin; XDR, extensively drug-resistant TB.

\section{Non-MDR outcomes}

Patients with mono or poly-DR-TB not meeting criteria for MDR were treated in the DR-TB programme using second-line drugs and were also eligible to participate in community or hospitalised care. Treatment regimens and durations varied according to resistance pattern and clinical status, with median treatment duration of 13 months (IQR 9-19 months). Among 148 patients with DR-TB who were initiated on treatment for DR-TB, $117(77 \%)$ were cured, $7(5 \%)$ completed, $5(3 \%)$ were lost to follow-up, $14(9 \%)$ died, $4(3 \%)$ failed, $3(2 \%)$ transferred out and $3(2 \%)$ remained on treatment at the time of data censoring. There was no significant difference in the proportion of each outcome according to community $(\mathrm{n}=79)$ or hospitalised status $(\mathrm{n}=74)$. One patient with treatment failure had initiated treatment for DR-TB but was found to have MDR after repeat DST; the patient was transitioned to MDR treatment and the outcome of that treatment episode was included in the cohort of MDR patients.

\section{Predictors of treatment success}

Predictors of treatment success were evaluated using logistic regression (table 3 ). The crude odds for treatment success were similar between the hospitalised and community groups, and this similarity persisted after adjusting for sex, age, HIV status, resistance pattern and
BMI (adjusted OR (aOR) 1.0 community vs hospitalised, $95 \%$ CI 0.63 to $1.60, \mathrm{p}=0.99$ ).

Factors strongly associated with decreased odds of treatment success included: age $\geq 44$, HIV infection and baseline BMI $<16$. While not statistically significant, the relative odds of treatment success for patients with XDR-TB were $31 \%$ compared with patients with INH and RIF resistance alone or RIF resistance diagnosed by GXP (table 3). The relative odds of treatment success were only $33 \%$ in HIV-infected patients compared with HIV-uninfected patients, a relationship that persisted after adjustment for potential confounders $(\mathrm{p}<0.001)$ (table 3). Patients with BMI less than 16 (severely underweight) at the time of treatment initiation were only $40 \%$ as likely to have treatment success compared with patients with a baseline $\mathrm{BMI} \geq 16$, after adjusting for confounders $(p<0.001)$. Older age was also significantly associated with lower odds of treatment success (aOR 0.40, p<0.001).

To reduce bias due to early in-hospital deaths in the comparison between community and hospitalised groups, the treatment cohort was restricted to patients who survived for at least 1 month after starting treatment in a sensitivity analysis. Twenty patients, including 11 HIV-infected patients (nine of whom were on ART), died in hospital less than 1 month after starting treatment, and were thus excluded from this sensitivity analysis. The aOR for treatment success in the community 
versus hospitalised groups was not different from the estimate from the entire cohort (aOR $0.86,95 \%$ CI 0.53 to 1.40 ), and this was still not statistically significant $(\mathrm{p}=0.55)$. ORs of all other predictors were similar.

\section{DISCUSSION}

The Cambodian MDR-TB programme achieved $77 \%$ treatment success (cure or completion) using a standardised treatment regimen between September 2006 and June 2015. Treatment success was achieved equally in patients with MDR-TB treated in hospitalised and community arms. Notably, this level of treatment success observed in the community arm is equivalent or superior to outcomes achieved in other resource-limited settings with prolonged initial inpatient hospitalisation, ${ }^{18}$ and equivalent or superior to other community-based MDR-TB treatment programmes. ${ }^{19}$

The CHC/NTP collaborative Cambodian MDR-TB programme represents the first large-scale MDR-TB treatment programme in Asia with a significant home-based component. The outcomes from the Cambodian MDR-TB programme demonstrate that a programme designed with the following fundamental components can achieve high rates of cure and completion in a resource-limited setting: (1) the option for primarily ambulatory, community-based care from the initiation of treatment; (2) developing a treatment programme on the foundation of an existing community-based TB treatment programme network; (3) coordination between NGO partners and the NTP in order to achieve programme sustainability within a national framework.

Successful community-based treatment for MDR-TB has been documented in other settings including Peru, ${ }^{20}$ with a primarily HIV-negative population, and South Africa and India, ${ }^{21-24}$ with primarily HIV-infected populations. In the Philippines, a high-MDR, low-TB/HIV coinfection burden country, the MDR-TB programme has successfully scaled up from a public-private partnership mix to integrate fully with the NTP framework. ${ }^{25}$ Cambodia, with 20\% HIV infection among the patients with MDR-TB enrolled in this cohort, represents an intermediate HIV scenario, to which the programme has adapted by building on a foundation of treatment: volunteer supporter supervised, community-based treatment for both TB and HIV, ensuring high levels of coverage in patients with MDR-TB (94\%) and close collaboration between HIV and MDR-TB clinicians. Other essential components of the Cambodian programme included community adherence support, home infection control support and ongoing expert clinical mentorship to programme clinicians. The Cambodian programme has since been successfully replicated in Ethiopia, with a close NGO-NTP relationship in the initiation of the programme, with similarly high rates of treatment success, and including a limited cohort of entirely homebased MDR-TB initiation by the NGO before the Ethiopian NTP expanded home initiation widely. ${ }^{26}$
The lack of difference between cure/completion outcomes in community and hospitalised treatment groups in the Cambodian programme highlights the feasibility and success of an approach that has numerous theoretical advantages. Although concerns about complicated medical management, patient loss to follow-up and transmission of MDR-TB have been cited as reasons to require hospitalisation for treatment,${ }^{27}$ Cambodian programme data demonstrate that outpatient initiation can be equally effective for some patients. Other evidence also supports this approach. In the Philippines, community-based care has been associated with decreased risk of MDR treatment loss to follow-up compared with patients required to stay at a centralised site. ${ }^{28}$ An ambulatory MDR-TB treatment programme in South Africa had superior outcomes to its hospitalised counterpart. ${ }^{23}$ The WHO now recommends ambulatory-based care with minimised clinic visits whenever possible and a recent meta-analysis comparing ambulatory with hospitalised MDR-TB care found equivalent treatment success between the models. ${ }^{16}{ }^{19}$ Cambodia's isolation bed capacity would be quickly overwhelmed if treatment required inpatient stays for all patients, and untreated, sick patients would remain in the community with no resources to minimise transmission of MDR-TB. By prioritising early access to appropriate MDR-TB treatment, home infection control and health staff capacity building for MDR-TB clinical management, hospital settings may be reserved for the sickest patients requiring inpatient clinical management or for those patients who do not have the social infrastructure allowing successful outpatient management. Significantly, outpatient therapy allows the patient and their caregivers to remain in their homes and within their social and family networks, making the long therapy more tolerable.

Despite overall high rates of cure and completion, the programme still experienced a death rate of $16 \%$ overall. HIV infection, older age and very low baseline BMI emerged as significant predictors of failure to achieve cure or completion. Of note, based on BMI criteria, patients treated in the Cambodian programme may have been more clinically ill than MDR patients in other settings, ${ }^{20}{ }^{28}$ which may in part account for the high mortality seen here, although it is still comparable to other MDR programmes. ${ }^{19}$ The decreased treatment success seen in HIV-infected patients, even those receiving ART, is consistent with the significant morbidity of MDR/HIV coinfection seen in resource-limited settings, though the $27 \%$ mortality among patients in this cohort is lower than a $38 \%$ pooled adult mortality estimate from 30 other studies. ${ }^{29}$

As an observational evaluation of community versus hospital-based MDR-TB treatment, our findings have some limitations. Patients were not randomised to hospital versus community-based treatment, and baseline characteristics of the two patient groups were indeed different, with patients in the hospitalised 
group more likely to have lower BMIs, more extensively resistant $\mathrm{TB}$ and possibly sicker, with more indications for prolonged hospitalisation. We controlled for some baseline differences in our analysis by adjusting for BMI, age, sex, resistance pattern and HIV status. In a sensitivity analysis, we restricted our analysis of outcomes to the subgroup of patients who survived the first month of treatment, in order to minimise this bias, and continued to find no statistically significant difference in outcome between the hospitalised and community-based groups. However, there were patients for whom early hospital discharge would be inappropriate, due to severe disease or comorbidities requiring inpatient management. Globally, throughout much of the reporting period, MDR-TB treatment frequently required prolonged inpatient hospital stays, regardless of the clinical status of the patient. We conclude from the Cambodian programme outcomes that for patients with MDR-TB who do not require hospital-level management of severe illness, excellent treatment outcomes can be achieved in the outpatient setting.

\section{CONCLUSIONS}

The Cambodian MDR-TB programme achieved excellent treatment outcomes with $77 \%$ treatment success over the first 10 years of the programme. Treatment outcomes among patients treated in the community after 30 days or less of hospitalisation were as successful as those among patients treated with prolonged hospitalisation. The global community is in increasing need of MDR-TB treatment approaches that do not rely on expensive, impractical hospital-based care that is both infeasible and results in the denial of treatment due to limited capacity in many settings. The Cambodian programme is a model, replicable programme in a moderate-HIV burden setting to implement such an approach. Innovations initiated in the field through community-based NGOs can successfully transition into a national programmatic setting as exemplified by the CHC-NTP MDR partnership in Cambodia.

Acknowledgements We gratefully acknowledge the patients and their families, the $\mathrm{CHC}$ and National Tuberculosis Program staff, and the communities whose dedication is the foundation for the treatment successes of the Cambodian MDR-TB programme and the NGO/NTP partnership. We thank Wallis Annenberg for her moral and financial support from the beginning of the programme in 2006, which allowed Cambodians to access high-quality MDR-TB care. We are grateful to the CHC mobile MDR nurse team including Chap Sophat, Kim Loern, Real Sarom, Touch Chhean and Nou Rom whose compassionate and devoted care, along with the MDR-TB treatment site physicians and nurses, is at the core of the success of this program. We thank the Cambodian national technical working group for MDR-TB for their ongoing support and especially we thank our colleagues from the MSF France and MSF Belgium teams for their solidarity and support and $\mathrm{Dr}$ Rajendra Prasad Yadav from WHO Cambodia for his support through the years. We are grateful to Didier Laureillard and F Xavier Blanc who provided critical help during the preparation of the first Green Light Committee application for MDR drugs on behalf of the CAMELIA trial (NCT01300481), the NIH and ANRS (ANRS1295/CIPRA KH001, NCT01300481) for their support of the first patients during the CAMELIA trial. Second-line drug support was received from UNITAID, the GFATM, Medecins San Fronteires (MSF France and MSF Belgium), USAID and Ministry of Health of Cambodia, and we thank Eli Lilly \& Co for the donation of capreomycin for select patients in the early years. We are indebted to the Holly
Myers and Kirk Neely, Jeanne Sullivan, Bud and Mimi Frankel, and Nancy and Steve Crown for their support.

Contributors Conception and design of the work: TS, SS, TEM, AES, AEG. Acquisition of data: SS, AES, TS, SKha, RS, SKhe, SC, SN, BK, PCS, HB, TEM Analysis and interpretation of data: AES, SS, SKhe, TEM, AEG.

Funding The CHC Cambodian MDR-TB Program was funded by a grant from the Annenberg Foundation (2006 onwards), grants from the Blue Oak and Frankel Foundations, and a gift from Nancy and Steve Crown. The program also received support from USAID/WHO (2012-2014), Global Fund for TB, Malaria and AIDS (GFATM) (2015), and USAID/FHI360 (2016).

Disclaimer The funders had no role in study design, data collection and analysis, decision to publish, or preparation of the manuscript.

Competing interests None declared.

Patient consent Not required.

Provenance and peer review Not commissioned; externally peer reviewed. Data sharing statement № additional data are available.

Open Access This is an Open Access article distributed in accordance with the Creative Commons Attribution Non Commercial (CC BY-NC 4.0) license, which permits others to distribute, remix, adapt, build upon this work non-commercially, and license their derivative works on different terms, provided the original work is properly cited and the use is non-commercial. See: http://creativecommons.org/ licenses/by-nc/4.0/

(C) Article author(s) (or their employer(s) unless otherwise stated in the text of the article) 2018. All rights reserved. No commercial use is permitted unless otherwise expressly granted.

\section{REFERENCES}

1. WHO. Global Tuberculosis Report 2017. Geneva: WHO, 2017.

2. WHO. Global tuberculosis report 2016. Geneva: World Health Organization, 2016.

3. WHO. Multidrug and extensively drug-resistant TB (M/XDR-TB): 2010 global report on surveillance and response. Geneva: WHO, 2010.

4. Tamura M, Eam KK, Kimura K, et al. National HIV prevalence surveillance among TB patients through periodic surveys: experience in Cambodia. Int J Tuberc Lung Dis 2008;12:20-5

5. Sar B, Keo C, Leng C, et al. Anti-tuberculosis drug resistance and HIV co-infection in Phnom Penh, Cambodia. Southeast Asian J Trop Med Public Health 2009;40:104-7.

6. Schnippel K, Rosen S, Shearer K, et al. Costs of inpatient treatment for multi-drug-resistant tuberculosis in South Africa. Trop Med Int Health 2013;18:109-16.

7. WHO. Guidelines for the programmatic management of drugresistant tuberculosis. Geneva: WHO, 2008.

8. Miles SH, Maat RB. A successful supervised outpatient shortcourse tuberculosis treatment program in an open refugee camp on the Thai-Cambodian border. Am Rev Respir Dis 1984;130:827-30.

9. Thim S, Sath S, Sina M, et al. A community-based tuberculosis program in Cambodia. JAMA 2004;292:566-8.

10. Voskens J, Yesudian M, Chak C, et al. Cambodia community DOTS and PPM evaluation report 2008. Phnom Penh, 2008.

11. Blanc FX, Sok T, Laureillard D, et al. Earlier versus later start of antiretroviral therapy in HIV-infected adults with tuberculosis. N Engl J Med 2011;365:1471-81.

12. WHO. The New Global Framework to support expansion of MDR-TB services and care. 2012 http://www.who.int/tb/challenges/mdr/ greenlightcommittee/en/.

13. Cambodian Ministry of Health. Annual TB Report 2011. Phnom Penh: Cambodian Ministry of Health, 2012.

14. Song R, Sam S, Cardenas V, et al. Novel diagnostic modalities for tb diagnosis among children in Cambodia. Int J Tuberc Lung Dis 2011; $15:$ S48

15. CENAT. Programmatic management of drug-resistant TB in Cambodia: Technical and Operational Guidelines. Phnom Penh: CENAT, 2011.

16. Falzon $\mathrm{D}$, Jaramillo $\mathrm{E}$, Schünemann $\mathrm{HJ}$, et al. WHO guidelines for the programmatic management of drug-resistant tuberculosis: 2011 update. Eur Respir J 2011;38:516-28.

17. WHO. Definitions and reporting framework for tuberculosis -2013 revision. Geneva: WHO/HTM/TB/2013.2, 2014.

18. Nathanson E, Lambregts-van Weezenbeek C, Rich ML, et al. Multidrug-resistant tuberculosis management in resource-limited settings. Emerg Infect Dis 2006:12:1389-97.

19. Bassili A, Fitzpatrick C, Qadeer E, et al. A systematic review of the effectiveness of hospital- and ambulatory-based management 
of multidrug-resistant tuberculosis. Am J Trop Med Hyg 2013;89:271-80

20. Mitnick C, Bayona J, Palacios E, et al. Community-based therapy for multidrug-resistant tuberculosis in Lima, Peru. N Engl J Med 2003;348:119-28.

21. Padayatchi N, Friedland G. Decentralised management of drugresistant tuberculosis (MDR- and XDR-TB) in South Africa: an alternative model of care. Int J Tuberc Lung Dis 2008;12:978-80.

22. Heller T, Lessells RJ, Wallrauch CG, et al. Community-based treatment for multidrug-resistant tuberculosis in rural KwaZulu-Natal, South Africa. Int J Tuberc Lung Dis 2010;14:420-6.

23. Loveday $\mathrm{M}$, Wallengren $\mathrm{K}$, Brust $\mathrm{J}$, et al. Community-based care vs. centralised hospitalisation for MDR-TB patients, KwaZulu-Natal, South Africa. Int J Tuberc Lung Dis 2015;19:163-71.

24. Isaakidis P, Cox HS, Varghese B, et al. Ambulatory multi-drug resistant tuberculosis treatment outcomes in a cohort of HIV-infected patients in a slum setting in Mumbai, India. PLoS One 2011;6:e28066.

25. Quelapio MI, Mira NR, Orillaza-Chi RB, et al. Responding to the multidrug-resistant tuberculosis crisis: mainstreaming programmatic management to the Philippine National Tuberculosis Programme. Int $J$ Tuberc Lung Dis 2010;14:751-7.

26. Meressa D, Hurtado RM, Andrews JR, et al. Achieving high treatment success for multidrug-resistant TB in Africa: initiation and scale-up of MDR TB care in Ethiopia--an observational cohort study. Thorax 2015;70:1181-8.

27. Brust JC, Shah NS, Scott M, et al. Integrated, home-based treatment for MDR-TB and HIV in rural South Africa: an alternate model of care. Int $J$ Tuberc Lung Dis 2012;16:998-1004

28. Gler MT, Podewils LJ, Munez N, et al. Impact of patient and program factors on default during treatment of multidrug-resistant tuberculosis. Int J Tuberc Lung Dis 2012;16:955-60.

29. Isaakidis P, Casas EC, Das M, et al. Treatment outcomes for HIV and MDR-TB co-infected adults and children: systematic review and meta-analysis. Int $J$ Tuberc Lung Dis 2015;19:969-78. 